\title{
Making the Design Process More Usable: Aligning Design with User Performance
}

\author{
Roberto K. Champney, Christina Kokini, and Kay M. Stanney \\ Design Interactive Inc., 1221 E. Broadway Suite 110, \\ Oviedo Fl 32765, United States \\ \{Roberto.Champney, Christina.Kokini, \\ Kay.Stanney\}@designinteractive.netx
}

\begin{abstract}
One key drawback when evaluating usability return on investment (ROI) is that the assessment criteria are often subjective, making it difficult for members of a development team to buy in to the need to support usabilityderived redesign recommendations. It is thus necessary to convey to the development team the importance of design for usability in a format that is universally understandable. The use of measurable usability requirements to assess usability ROI was found to be an effective approach to align design with operational performance and at the same time justify the need for redesign to the development team. This approach should result in better development team cohesion, as well as superior end product performance, which captures and supports the needs of end users and other stakeholders alike. In the current effort, this alignment process is described, and the utility of the approach is demonstrated by its application in a field case study of the successful design of a software application.
\end{abstract}

Keywords: Usability, Return on Investment, User Experience, Design.

\section{Introduction}

The importance of usability, in particular the importance of including user-centered evaluations from the beginning of the design lifecycle, has been common knowledge to human factors professionals for years [1]. However, other stakeholders, including members of the development team, often see such "soft" assessments as a hassle or even a luxury. While a number of studies have documented the value of usability, in practice [2] it is often necessary to continuously demonstrate the return on investment (ROI) such activities have to the overall development effort. One key drawback when evaluating usability ROI is that the assessment criteria are often subjective, which makes it difficult for members of a development team to buy into the need to support usability-derived redesign recommendations. It is thus necessary to convey to the development team the importance of design for usability in a format that is universally understandable. In the current effort, this process is described, and the utility of the approach is demonstrated by its application in a field case study of the successful design of a software application. 


\section{Background}

Design and development is a multi-disciplinary effort that must take into consideration the input from the multiple disciplines involved. This often involves an iterative approach where designs are evaluated by usability practitioners alone or with end-users and result in the identification of usability shortcomings (i.e., issues). These evaluations are formative in nature [3] and thus are diagnostic, iterative, and focus on identifying usability problems that require resolution before a design is released for widespread use by its intended user population [4,5]. This is in contrast to summative evaluations which focus on quantifying usability performance. In ideal conditions, these findings would be readily transformed into design recommendations that would be instantiated into the system under review. Yet given the multi-disciplinary nature of development teams, the identified usability issues must often be carefully communicated to the other members of the team before they can be addressed so that they can understand them and also because usability issues must compete with a broad range of other priorities (e.g., time and budget, project goals, technical challenges). This creates a challenge since such reports must convey the importance of the findings in a way that other team members can understand and relate to such that they will include usability issues as a top priority.. In such an environment it becomes imperative that usability practitioners develop means to convey the criticality of their findings and align such findings to tangible metrics of performance. The National Institute of Standards and Technology (NIST) recognizes this as described in their Industry USability Reporting (IUSR) Formative Project [6] where they state "...With many more techniques available for this type of work, practitioners need clear definitions goals and guidelines to use in planning and conducting formative evaluations, especially in communicating/reporting the work (and its value) to colleagues and customers." (pg. 3). This is particularly important because often times it is the software development and management components of the team who assess the validity and criticality of usability findings, and not the usability practitioner [7].

For usability practitioners, this challenge is at the heart of their value proposition and thus several approaches have been suggested in the past to help address it. Formatting guidelines have been a popular approach which suggests that usability reports should be formatted for their intended audience. More specifically, reporting guidelines prescribe what elements or characteristics a report should include (e.g., drafting short-reports, an executive summary, usability issue severity classification, frequency of issues, positive findings, professional layout) [8]. Other strategies are more involved and prescribe the indoctrination of other stakeholders (e.g., the development team) into the value of the usability process in order to develop empathy for usability issues [7, 9]. These approaches involve the participation of the development team during user interviews, field visits, user testing, etc. or in exercises involving the prioritization of observed usability issues in order to develop a shared mental model of users' needs. While such approaches are of great value towards increasing the effectiveness of communicating usability findings and promoting the redesign of a system, they may be too time- and resource-intensive for the development team. These approaches also lack the ability to measure and convey the system's level of success from a usability perspective and are not prescriptive towards 
which issues affect project goals set by the entire development team. In addition, such approaches still do not provide sufficient insight to decision makers in the development team who may not be intimately familiar with the development work taking place, but who have direct input into the direction of an effort. In order to be more effective, usability evaluation reports need to incorporate objective, measureable operational objectives that can be tied directly back to project goals.

\section{Proposed Approach}

This work presents an alternative approach to supplement the reporting guidelines proposed by earlier work. The proposed approach incorporates the definition and conveyance of usability objectives that are measureable and aligned with project goals. The usability alignment process follows the traditional development lifecycle, including best practices for user-centered design, yet there are key differences to supplement and emphasize the focus on aligning usability needs to design elements, ensuring that project goals are met.

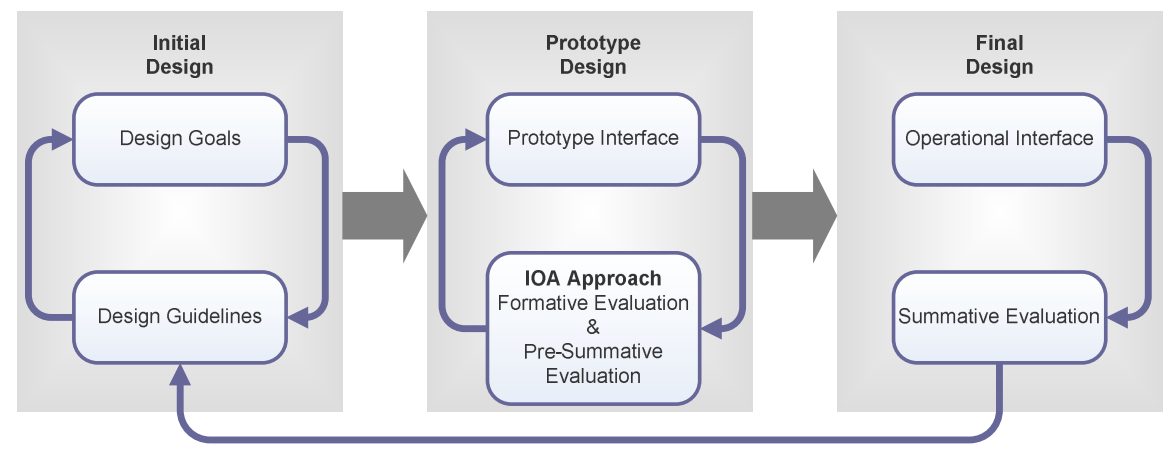

Fig. 1. Iterative Operational Assessment (IOA) Approach

What is proposed herein is an Iterative Operational Assessment (IOA) approach, as illustrated in Figure 1, which takes place iteratively during the development of a tool or system before it culminates in a final operational design. Traditionally formative usability evaluations take place once a prototype design has been developed, and summative usability evaluations don't take place until a final design is ready [10]. The IOA approach similarly follows this method, yet it incorporates "pre-summative" evaluations at the prototype stage as illustrated in Figure 1. The term "pre-summative" is coined here in reference to the assessment of human performance to evaluate the capabilities of the system at the prototype stage. Such an approach allows the design to be measured against an objective target (i.e., an operational objective linked to the project's goals) iteratively such that deviations from a desired outcome can be addressed via redesign. In order to derive value from these pre-summative evaluations, the measures to be assessed must be carefully thought through and agreed upon by the development team. This process is described next and is followed by a case study describing how this approach has been successfully applied in the field. 


\section{The Iterative Operational Assessment (IOA) Approach}

The IOA approach is composed of four steps, as illustrated in Figure 2, where the outcome of one step is fed into the next as described below.

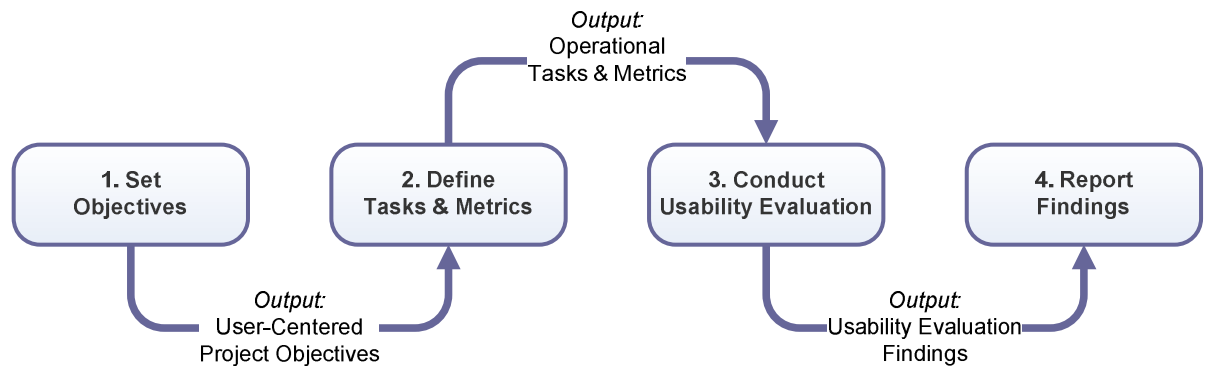

Fig. 2 The four steps of the IOA Approach

\subsection{Set Objectives}

As with traditional approaches, the process begins with the identification of relevant stakeholders, including decision makers, the development team and the usability practitioner as an advocate of the end-user. It is especially important to include decision makers that can identify the key project goals. The objective of this step is to provide alignment between project goals and user needs, and not to allow for decision makers to dictate design. One should always maintain user-centered vigilance throughout the process,yet it is necessary to obtain buy-in and insight from decision makers and other stakeholders. In this step (Table 1), the practitioner facilitates the identification of project goals via the application of user analysis methods with stakeholders (e.g., meetings, interviews, review of documentation). These goals are then decomposed into user-centered objectives (UC-Objectives) informed by the contextual field and task analyses that should have taken place earlier (as part of any usability engineering effort). Once the user-centered objectives have been defined, prioritized and agreed upon by stakeholders, it is time to move to the next step and define measures of expectation.

Lessons learned from the application of this method suggest that this approach ensures that decision makers are provided with data they understand and have agreed to, and thus eases any skepticism that may exist. Redesign recommendations

Table 1. Step 1 - Set Objectives

\begin{tabular}{ll}
\hline Process Component & \multicolumn{1}{c}{ Items } \\
\hline Inputs & Task and Domain Analysis, Project Scope and Objectives \\
Outputs & User-Centered Project Objectives \\
Participants & $\begin{array}{l}\text { Decision Makers, Development Team, Usability } \\
\text { Practitioner }\end{array}$ \\
\hline
\end{tabular}


associated with operational objectives (the UC-Objectives) set by the decision makers (as well as the rest of the stakeholders) are more likely to have more leverage during a more comprehensive prioritization of design requirements (i.e., design functionalities to support stakeholders', including end-users' tasks and goals) later in the process.

\subsection{Define Tasks and Metrics}

With the UC-Objectives (e.g., increase efficiency of field personnel) agreed upon on the prior step, it is now necessary to derive operational tasks and measures (Table 2) that would assess such objectives. In this step each UC-Objective is decomposed into related operational tasks (e.g., completing tasks $\mathrm{X}$ and $\mathrm{Y}$ ) with the insight of the task and domain analysis work that should be part of any usability engineering effort. For each of these operational tasks, appropriate metrics (e.g., time to complete task) are developed such that performance by the system on these tasks can be objectively assessed.

Lessons learned suggest that these test tasks should be designed to achieve the same contextual objectives (e.g., select a training scenario that matches a prescribed training objective) as the baseline condition (i.e., prior to the design) in order to ensure objective comparisons.

Table 2. Step 2 - Define Tasks and Metric

\begin{tabular}{ll}
\hline Process Component & \multicolumn{1}{c}{ Items } \\
\hline Inputs & Task and Domain Analysis, User-Centered Project \\
& Objectives \\
Outputs & Operational Tasks and Metrics \\
Participants & Usability Practitioner \\
\hline
\end{tabular}

\subsection{Usability Evaluation}

The operational tasks and metrics defined in the prior step provide the guidance necessary to plan for a customary usability evaluation as. The key difference is that the tasks identified are now aligned not only with user needs, but also with project goals. Thus these contextually-relevant tasks become test tasks that can be used for iterative usability evaluations (Table 3 ). These evaluations can then assess system performance in the baseline condition (i.e., the way tasks are currently completed without the system being designed) and with each iteration of the system design. This does not imply that only user testing is conducted, in fact other evaluation methods should be utilized in conjunction, and the tasks identified in step 2 should be used to organize and prioritize the findings. A key difference in the way usability evaluations are conducted with the IOA approach is the "pre-summative" part of the evaluation. This is a stricter form of user testing than the traditional formative user testing since it requires a larger sample in order to provide a more reliable assessment of user performance. It is also not a true summative evaluation since the purpose is not to qualify the system to a desired level of performance, nor is the desired to achieve statistical significance, but to guide the design of the system such that it ultimately reaches a desired level of performance. 
Lessons learned suggest that incorporating quantifiable usability requirements at this stage of the development lifecycle allows for the objective evaluation of the design's usability in terms of the key identified and agreed upon UC-objectives.

Table 3. Step 3 - Conduct Iterative Usability Evaluations

\begin{tabular}{ll}
\hline Process Component & \multicolumn{1}{c}{ Items } \\
\hline Inputs & Operational Tasks and Metrics \\
Outputs & Usability Evaluation Findings \\
Participants & Usability Practitioner, End-Users \\
\hline
\end{tabular}

\subsection{Report Findings}

The final step in the IOA approach is to report the findings such that the identified usability issues can be addressed. This is done in coordination with other formative usability evaluation methods which together with the quantifiable usability performance allow for definitive justification and prioritization of usability-derived changes to the design based on their impact. The reporting of the findings is done with input from available guidelines and tailored for their intended audience (e.g., the development team). The key contribution of the IOA approach is in how information is presented and prioritized. Given the alignment with the project goals,one can present the findings and redesign recommendations aligned with these goals in order to provide weight to their criticality. Further, objective comparison with the baseline and across design iterations provides a tangible indication to all members of the development team as to the capabilities of the design and reasons behind any redesign recommendations, thus providing a universal language to support the necessary communication in a multidisciplinary development team. Such alignment not only allows a development team to design and test iteratively for an objective performance target, but it also allows them to understand the progression of the design from a usability perspective.

\section{Case Study}

The IOA approach to formative usability evaluations has been applied in the field and the results of this real-life project are presented next as a proof-of-concept.

\subsection{Background}

During a recent project, the authors applied the IOA approach to a system being design to address the needs of the United States Marine Corps (USMC). The increases in training demands faced by today's Marines require that they take on more roles and master more knowledge, skills, and attitudes than ever before. Not only are more strenuous training requirements being levied, but at the same time the military is faced with the need to get troops trained with fewer resources. As a result, there is a need to increase the efficiency and effectiveness of learning to compress learning time and at the same time decrease instructor time requirements. The Deployable Virtual 
Training Environment (DVTE) has been supplied as a potential solution to the challenge; it comprises a suite of laptop computers infused with a variety of training programs and simulation applications. Yet challenges still exist. To address some of the remaining challenges faced by the USMC, the Instruction Support Station (ISS) was designed and developed utilizing the IOA approach. Its purpose is to assist instructors using the DVTE system to conduct training. The application of this approach to the development of the ISS is discussed next.

\subsection{Set Objectives}

During the development effort of the ISS, the authors conducted a task analysis of the Marine training environment as is customary of human-computer interaction projects. This data was then utilized during multiple meetings with stakeholders, including decision makers and the multi-disciplinary development team to explicitly define key operational objectives for the project (i.e., the UC-Objectives). Two objectives are presented here: 1) Enhance instructor efficiency and 2) Enhance instructional effectiveness. These objectives did not only helpe define the theme of the effort but provided a means to prioritize the design effort into those design capabilities that would support these objectives.

\subsection{Define Tasks and Metrics}

Once the objectives had been defined and agreed upon, the authors, together with members of the development team, decomposed these objectives into operational tasks. Two examples are presented here: 1) Decrease the time to setup a training operation, and 2) Increase the ability to find suitable scenarios matching a desired training objective. With these operational tasks defined it was now possible to further define metrics to assess performance on these tasks, in this case: 1) time on task, and 2) whether or not a designated scenario was chosen given a particular training objective.

\subsection{Usability Evaluation}

The tasks and metrics defined were then integrated into the usability evaluation protocol that was used during the effort. As part of the user-centered design approach that took place, iterative design of low fidelity prototypes and multiple iterations of working prototypes were developed and evaluated. Both heuristic and empirical usertesting evaluations of the designs took place, allowing the authors to assess usability issues of the designs and address the issues via redesign recommendations. While the evaluations covered all of the aspects of the ISS design, the pre-summative user testing was focused on the key tasks defined and assessed via the metrics from the prior step. Together the findings of the evaluations were then integrated and reported.

\subsection{Report Findings}

In reporting the findings, guidance from best-practices was incorporated and integrated into redesign recommendations. The main difference from the traditional reporting approach was with the use of the pre-summative data. This data served to 
highlight current performance and to press upon the development team the need to implement the redesign recommendations pertaining to those key functionalities that affected the tasks representative of the project goals.

\section{Percent Improvement in Setup Time}

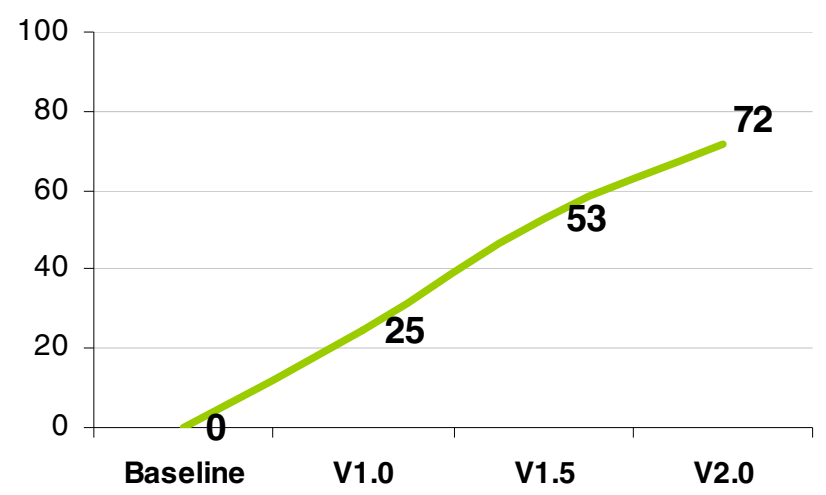

Fig. 2. Percent improvement in the setup time of a training operation from baseline

\section{Percent Improvement in Scenario Selection}

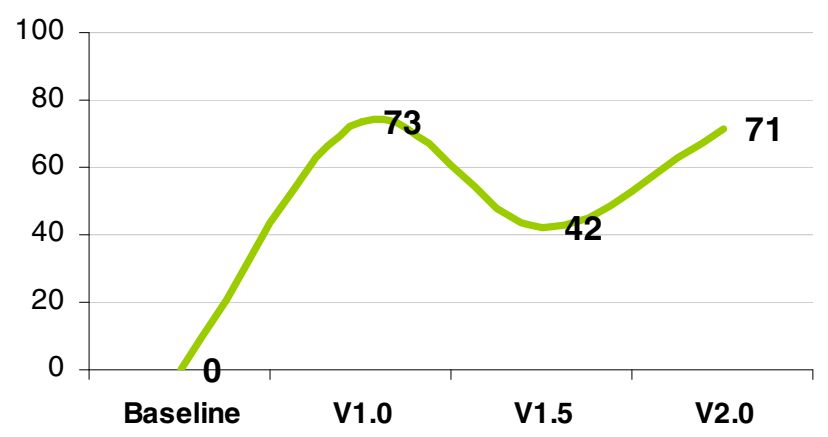

Fig. 3. Percent improvement in the selection of a designated scenario from baseline

\section{Discussion}

Through the iterative application of the IOA approach, the ISS design was guided to increase performance along those key tasks aligned with the project goal. In its 
current prototype version, the ISS has been able to achieve an improvement of $72 \%$ increase in efficiency of launching scenarios, and a $71 \%$ increase in the accuracy of selecting suitable scenarios for training. The authors found the IOA approach very powerful in quantifying the impact of the redesign recommendations. As shown in Figures 2 and 3 , the iterative evaluations and the prioritization of redesign recommendations were necessary in order to maximize their impact on the project goals. One interesting observation is the potential interaction effects that may be observed during this process. During the redesign of the ISS V1.5, several features were integrated in order to increase the performance of one objective (see Figure 2) resulting in repercussions towards a second objective (see Figure 3). Yet though the iterative process of continuous improvement via redesign, performance was regained and expected to increase as the design further matures.

\section{Conclusion}

In sum, the use of quantifiable user-centered objectives to assess usability ROI was found to be an effective approach to align design with operational performance and at the same time justify to the development team the reasons for the need to redesign. This approach should result in better development team cohesion, as well as superior end-product performance, which captures and supports the needs of end-users and other stakeholders alike.

Acknowledgement. This material is based upon work supported in part by the Office of Naval Research (ONR) under contract N00014-08-C-0186. Any opinions, findings and conclusions or recommendations expressed in this material are those of the authors and do not necessarily reflect the views or the endorsement of ONR.

\section{References}

1. Mayhew, D.J.: The usability engineering lifecycle. Morgan Kaufmann Publishers, Inc., San Francisco (1999)

2. Bias, R.G., Mayhew, D.J.: Cost-justifying Usability. Morgan Kaufmann Publishers, Inc., San Francisco (2005)

3. Hewett, T.T.: The role of iterative evaluation in designing systems for usability. In: Harrison, M.D., Monk, A.F. (eds.) People \& Computers: Designing for usability. Proceedings of the second conference of the BCS HCI specialist group, Cambridge University Press, Cambridge (1986); In: Booth, P.: An Introduction to Human-Computer Interaction, LEA, Hove, East Sussex, U.K. (1990)

4. Hartson, H.R., Andre, T.S., Williges, R.C.: Criteria for evaluating usability evaluation methods. International Journal of Human-Computer Interaction 13(4), 373-410 (2001)

5. Quesenbery, W.: Defining a summative usability test for voting systems: A report from the UPA 2004 Workshop on Voting and Usability (Online). Usability Professional's Association (2004),

http://www.usabilityprofessionals.org/upa_projects / voting_and_usability/documents/voting_summative_test.pdf (retrieved January 20, 2008) 
6. Theofanos, M., Quesenbery, W., Snyder, C., Dayton, D., Lewis, J.: Reporting on Formative Testing: A UPA 2005 Workshop Report. Usability Professionals Association (2005),

http: //www.usabilityprofessionals.org/usability_resources/ conference/2005/formative/\%20reporting-upa2005.pdf

7. Kneifel, A.A., Guerrero, C.: Using participatory inquiry in usability analysis to align a development team's mental model with its users' needs. In: Proceedings of the Society for Technical Communication 50th annual conference, Dallas, Texas, USA, May 18-21 (2003)

8. Dumas, J.S., Redish, J.C. A practical guide to usability testing, 2nd edn. Intellect, Portland, OR (1999)

9. Redish, J., Bias, R.G., Bailey, R., Molich, R., Dumas, J., Spool, J.M.: Usability in Practice: Formative usability evaluations - evolution and revolution. In: Proceedings of the Computer Human Interaction Conference, Minneapolis, Minnesota, USA, April 20-25 (2002)

10. Kies, J.K., Williges, R.C., Rosson, M.B.: Coordinating computer-supported cooperative work: A review of research issues and strategies. Journal of the American Society for Information. Science 49, 776-779 (1998) 